\title{
Research Article \\ Guaranteed Cost Control Design of 4D Lorenz-Stenflo Chaotic System via T-S Fuzzy Approach
}

\author{
Yi-You Hou, Meei-Ling Hung, and Jui-Sheng Lin \\ Department of Electrical Engineering, Far East University, Tainan 74448, Taiwan \\ Correspondence should be addressed to Yi-You Hou, yyhou@cc.feu.edu.tw
}

Received 12 January 2012; Accepted 12 March 2012

Academic Editor: Recai Kilic

Copyright (C) 2012 Yi-You Hou et al. This is an open access article distributed under the Creative Commons Attribution License, which permits unrestricted use, distribution, and reproduction in any medium, provided the original work is properly cited.

This paper investigates the guaranteed cost control of chaos problem in 4D Lorenz-Stenflo (LS) system via Takagi-Sugeno (T-S) fuzzy method approach. Based on Lyapunov stability theory and linear matrix inequality (LMI) technique, a state feedback controller is proposed to stabilize the $4 \mathrm{D}$ Lorenz-Stenflo chaotic system. An illustrative example is provided to verify the validity of the results developed in this paper.

\section{Introduction}

Chaos phenomenon which is a deterministic nonlinear dynamical system has been generally developed over the past two decades, based on its particular properties, such as broadband noise-like waveform, and depending sensitively on the system's precise initial conditions, and so forth. Due to its powerful applications in engineering systems, both control and synchronization/stability problems have extensively been studied in the past decades for chaotic systems. Recently, many papers studied the hyperchaotic system, and some dynamical behaviors are studied, such as Chen's system [1], Lorenz-Stenflo system [2], Josephson junctions [3], cell neural network [4], Lü system [5, 6], and Genesio System [7]. Several control schemes for the stability/synchronization/solution problem of nonlinear systems have been studied extensively, such as backstepping design [8], feedback control [9], adaptive control [10], intermittent control [11], fuzzy model based [12], and multistep differential transform [13]. On the other hand, Takagi-Sugeno (T-S) fuzzy concept was introduced by the pioneering work of Takagi and Sugeno and has been successfully and effectively used in complex nonlinear systems [14]. The main feature of T-S fuzzy model is that a nonlinear 
system can be approximated by a set of T-S linear models. The overall fuzzy model of complex nonlinear systems is achieved by fuzzy "blending" of the set of T-S linear models. Therefore, the controller design and the stability analysis of nonlinear systems can be analyzed via T-S fuzzy models and the so-called parallel distributed compensation (PDC) scheme [15-18].

Inspired by the researches mentioned above, this paper examines the problem of stability for the 4D Lorenz-Stenflo systems. To achieve this goal, based on the Lyapunov stability theory, PDC scheme, and the LMI optimization technique, a controller is derived to guarantee stability of the 4D Lorenz-Stenflo system. Finally, an example is given to illustrate the usefulness of the obtained results.

\section{Problem Formulation and Main Results}

A 4D Lorenz-Stenflo chaotic system is expressed by the following differential equation [2]:

$$
\begin{aligned}
& \dot{x}_{1}(t)=a\left(x_{2}(t)-x_{1}(t)\right)+b x_{4}(t), \\
& \dot{x}_{2}(t)=c x_{1}(t)-x_{1}(t) x_{3}(t)-x_{2}(t), \\
& \dot{x}_{3}(t)=x_{1}(t) x_{2}(t)-d x_{3}(t), \\
& \dot{x}_{4}(t)=-x_{1}(t)-a x_{4}(t),
\end{aligned}
$$

where $x_{1}, x_{2}, x_{3}, x_{4}$ are state variables and $a, b, c, d$ are called the Prandel number, rotation number, Rayleigh number, and geometric parameter of the system, respectively [2]. To investigate the control design of system (2.5), let the system's state vector $x(t)=$ $\left[\begin{array}{llll}x_{1}(t) & x_{2}(t) & x_{3}(t) & x_{4}(t)\end{array}\right]^{T}$ and the control input vector $u(t)$. Then, the state equations of $4 \mathrm{D}$ Lorenz-Stenflo chaotic system (2.1) can be represented as follows:

$$
\dot{x}(t)=A(x(t)) x(t)+B u(t),
$$

where

$$
A(x(t))=\left[\begin{array}{cccc}
-a & a & 0 & b \\
c & -1 & -x_{1}(t) & 0 \\
0 & x_{1}(t) & -d & 0 \\
-1 & 0 & 0 & -a
\end{array}\right]
$$

and $B$ is known constant matrix with appropriate dimensions.

The aim of this paper is to stabilize 4D Lorenz-Stenflo chaotic systems using T-S fuzzy controller. The continuous fuzzy system was proposed to represent a nonlinear system [14]. The system dynamics (2.2) can be captured by a set of fuzzy rules which characterize local correlations in the state space. Each local dynamic described by the fuzzy IF-THEN rule has 
the property of linear input-output relation. Based on the T-S fuzzy model concept, a general class of T-S fuzzy 4D Lorenz-Stenflo chaotic systems is considered as follows

\section{Model Rule i}

If $z_{1}(t)$ is $M_{i 1}$ and $\ldots z_{r}(t)$ is $M_{i r}$, then

$$
\dot{x}(t)=A_{i} x(t)+B_{i} u(t),
$$

where $z_{1}(t), z_{2}(t), \ldots, z_{r}(t)$ are known premise variables, $M_{i j}, i \in\{1,2, \ldots, m\}, j \in$ $\{1,2, \ldots, r\}$ is the fuzzy set, and $m$ is the number of model rules; $x(t)$ is the state vector and $u(t)$ is input vector. The matrices $A_{i}$ and $B_{i}$ are known constant matrices with appropriate dimensions. Given a pair of $(x(t), u(t))$, the final outputs of the fuzzy system are inferred as follows:

$$
\begin{aligned}
\dot{x}(t) & =\frac{\sum_{i=1}^{m} w_{i}(z(t)) \cdot\left\{A_{i} x(t) B_{i} u(t)\right\}}{\sum_{i=1}^{m} w_{i}(z(t))} \\
& =\sum_{i=1}^{m} \eta_{i}(z(t)) \cdot\left\{A_{i} x(t)+B_{i} u(t)\right\},
\end{aligned}
$$

where $z(t)=\left[z_{1}(t) z_{2}(t) \cdots z_{r}(t)\right], w_{i}(z(t))=\prod_{j=1}^{r} M_{i j}\left(z_{j}(t)\right), \eta_{i}(z(t))=w_{i}(z(t)) /$ $\sum_{i=1}^{m} w_{i}(z(t))$. The term $M_{i j}\left(z_{j}(t)\right)$ is the grade of membership of $z_{j}(t)$ in $M_{i j}$. In this paper, we assume that $w_{i}(z(t)) \geq 0, i \in\{1,2, \ldots, m\}$, and $\sum_{i=1}^{m} w_{i}(z(t))>0$. Therefore, we have $\eta_{i}(z(t)) \geq 0, i \in\{1,2, \ldots, m\}$ and $\sum_{i=1}^{m} \eta_{i}(z(t))=1$, for all $t \geq 0$.To derive the main results, we first introduce the cost function of system (2.4) as follows:

$$
J=\int_{0}^{\infty}\left[x^{T}(s) \cdot Q \cdot x(s)+u^{T}(s) \cdot R \cdot u(s)\right] d s,
$$

where $Q$ and $R$ are two given positive definite symmetric matrices. Associated with cost function (2.6), the fuzzy guaranteed cost control is defined as follows.

Definition 2.1. Consider the T-S fuzzy system (2.4); if there exist a control law $u(t)$ and a positive scalar $J^{*}$ such that the closed-loop system is stable and the value of cost function (2.6) satisfies $J \leq J^{*}$, then $J^{*}$ is said to be a guaranteed cost and $u(t)$ is said to be a guaranteed cost control law for the T-S fuzzy 4D Lorenz-Stenflo chaotic systems (2.4).

This paper aims at designing a guaranteed cost control law for the asymptotic stabilization of the T-S fuzzy 4D Lorenz-Stenflo chaotic systems (2.4). To achieve this control goal, we utilize the concept of PDC [14] scheme and select the fuzzy guaranteed cost controller via state feedback as follows.

\section{Control Rule j}

If $z_{1}(t)$ is $M_{j 1}$ and $\ldots z_{r}(t)$ is $M_{j r}$, then

$$
u(t)=-K_{j} x(t), \quad t \geq 0,
$$


where $K_{j}, j \in\{1,2, \ldots, m\}$ are the state feedback gains. Hence, the overall state feedback control law is represented as follows:

$$
u(t)=-\sum_{j=1}^{m} \eta_{j}(z(t)) \cdot K_{j} x(t), \quad t \geq 0
$$

Before proposing the main theorem for determining the feedback gains $K_{j}(j=1,2, \ldots, m)$, a lemma is introduced.

Lemma 2.2 (see [19] (Schur complement)). For a given matrix $S=\left[\begin{array}{ll}S_{11} & S_{12} \\ S_{12}^{T} & S_{22}\end{array}\right]$ with $S_{11}=S_{11}^{T}$, $S_{22}=S_{22}^{T}$, then the following conditions are equivalent:
(1) $S<0$,
(2) $S_{22}<0, S_{11}-S_{12} S_{22}^{-1} S_{12}^{T}<0$.

Now we present an asymptotic stabilization condition for T-S fuzzy 4D Lorenz-Stenflo chaotic systems (2.4).

Theorem 2.3. If there exist some positive definite symmetric matrices $\widehat{P}$ and matrices $\widehat{K}_{j}, j \in$ $\{1,2, \ldots, m\}$ such that the following $L M I$ condition holds for all $i, j \in\{1,2, \ldots, m\}$ :

$$
\widetilde{\Phi}_{i j}=\left[\begin{array}{ccc}
A_{i} \widehat{P}+\widehat{P} A_{i}^{T}-B_{i} \widehat{K}_{j}-\widehat{K}_{j}^{T} B_{i}^{T} & \widehat{P} & \widehat{K}_{j}^{T} \\
* & -Q^{-1} & 0 \\
* & * & -R^{-1}
\end{array}\right]<0 .
$$

Then system (2.4) is asymptotically stabilizable by controller (2.8). The stabilizing feedback control gain is given by $K_{j}=\widehat{K}_{j} \widehat{P}^{-1}$, and the system performance (2.6) is bounded by

$$
J \leq J^{*}=x^{T}(0) P x(0),
$$

where $P=\widehat{P}^{-1}$.

Proof. Define the Lyapunov functional:

$$
V(x(t))=x^{T}(t) P x(t),
$$


where $V(x(t))$ is a legitimate Lyapunov functional candidate and $P$ is positive definite symmetric matrices. By the system (2.4) with $\sum_{i=1}^{m} \eta_{i}(z(t))=1$, the time derivatives of $V(x(t))$, along the trajectories of system (2.4) with (2.6) and (2.8), satisfy

$$
\begin{aligned}
& \dot{V}(x(t))-\sum_{i=1}^{m} \sum_{j=1}^{m} \eta_{i}(z(t)) \eta_{j}(z(t))\left\{x^{T}(t)\left(Q+K_{j}^{T} R K_{j}\right) x(t)\right\} \\
& =\sum_{i=1}^{m} \sum_{j=1}^{m} \eta_{i}(z(t)) \eta_{j}(z(t))\left\{x^{T}(t)\left(P A_{i}+A_{i}^{T} P-K_{j}^{T} B_{i}^{T} P-P B_{i} K_{j}-Q-K_{j}^{T} R K_{j}\right) x(t)\right\} \\
& \leq \sum_{i=1}^{m} \sum_{j=1}^{m} \eta_{i}(z(t)) \eta_{j}(z(t)) x^{T}(t) \Phi_{i j} x(t) .
\end{aligned}
$$

In order to guarantee $\dot{V}(x(t))-\sum_{i=1}^{m} \sum_{j=1}^{m} \eta_{i}(z(t)) \eta_{j}(z(t))\left\{x^{T}(t)\left(Q+K_{j}^{T} R K_{j}\right) x(t)\right\}<0$, we need to satisfy $\Phi_{i j}<0$. By Lemma 2.2 (Schur complement) [19], and premultiplying and postmultiplying the $\Phi_{i j}$ in (2.12) by $P^{-1}>0, \Phi_{i j}<0$ are equivalent to $\widetilde{\Phi}_{i j}<0$ in (2.9), then we can obtain the following:

$$
\begin{aligned}
\dot{V}(x(t)) & \leq-\sum_{i=1}^{m} \sum_{j=1}^{m} \eta_{i}(z(t)) \eta_{j}(z(t)) x^{T}(t)\left(Q+K_{j}^{T} R K_{j}\right) x(t) \\
& =-\left(x^{T}(t) \cdot Q \cdot x(t)+u(t) \cdot R \cdot u(t)\right)<0 .
\end{aligned}
$$

From the inequality (2.13), $\dot{V}(x(t))<0$, we conclude that system (2.4) with (2.6) is asymptotically stable. Integrating (2.13) from 0 to $\infty$, we have

$$
\int_{0}^{\infty} \dot{V}(x(s)) d s=\lim _{t \rightarrow \infty} V(x(t))-V(x(0)) \leq-\int_{0}^{\infty}\left[x^{T}(s) \cdot Q \cdot x(s)+u^{T}(s) \cdot R \cdot u(s)\right] d s .
$$

Since that the system (2.4) with (2.6) is asymptotically stable, we can obtain the following results:

$$
\lim _{t \rightarrow \infty} V(x(t))=0
$$

Consequently, $J=\int_{0}^{\infty}\left[x^{T}(s) \cdot Q \cdot x(s)+u^{T}(s) \cdot R \cdot u(s)\right] d s \leq x^{T}(0) P x(0)=V(x(0))=J^{*}$. This completes the proof. 


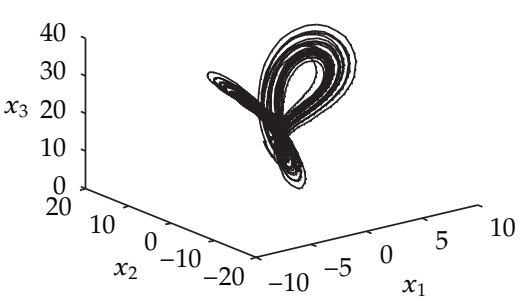

(a)

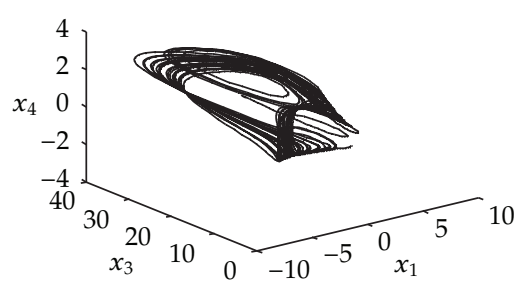

(c)

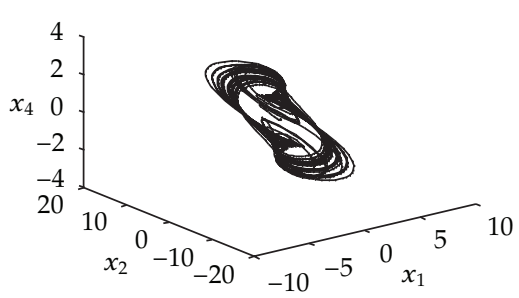

(b)

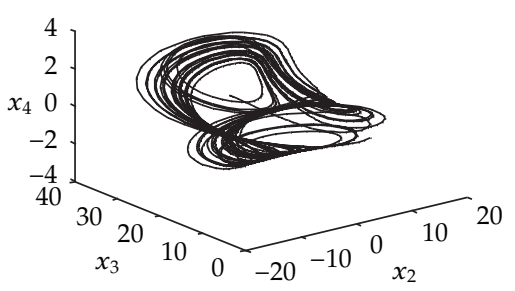

(d)

Figure 1: The chaotic attractor of the 4D Lorenz-Stenflo chaotic system.

\section{Numerical Simulation and Analysis}

In this section, a numerical example is presented to demonstrate and verify the performance of the proposed results. Consider a $4 \mathrm{D}$ Lorenz-Stenflo as given in (2.1) with the following parameters [2]: $a=1.0, b=1.5, c=26$, and $d=0.7$.

From the simulation result, we can get that $x_{1}(t)$ is bounded in interval [ -77$]$. By solving the equation, $M_{1}$ and $M_{2}$ are obtained as follows:

$$
M_{1}\left(x_{1}(t)\right)=\frac{1}{2}\left(1+\frac{x_{1}(t)}{7}\right), \quad M_{2}\left(x_{1}(t)\right)=1-M_{1}\left(x_{1}(t)\right)=\frac{1}{2}\left(1-\frac{x_{1}(t)}{7}\right) .
$$

$M_{1}$ and $M_{2}$ can be interpreted as membership functions of fuzzy sets. Using these fuzzy sets, the nonlinear system with time-varying delays can be expressed by the following T-S fuzzy models.

Rule 1. If $x_{1}(t)$ is $M_{1}$, then

$$
\dot{x}(t)=A_{1} x(t)+B_{1} u(t),
$$

Rule 2. IF $x_{1}(t)$ is $M_{2}$, then

$$
\dot{x}(t)=A_{2} x(t)+B_{2} u(t),
$$




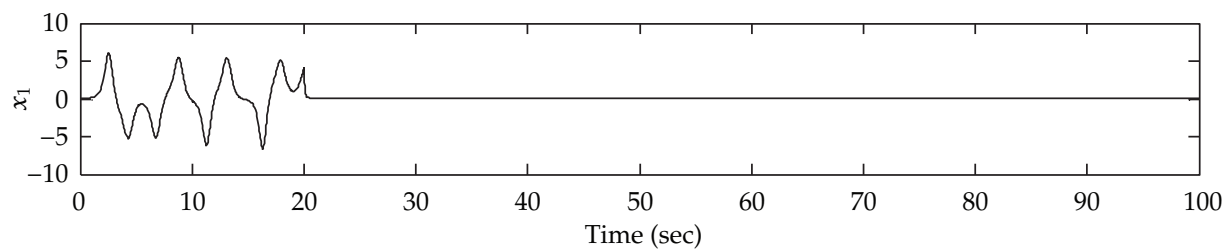

(a)

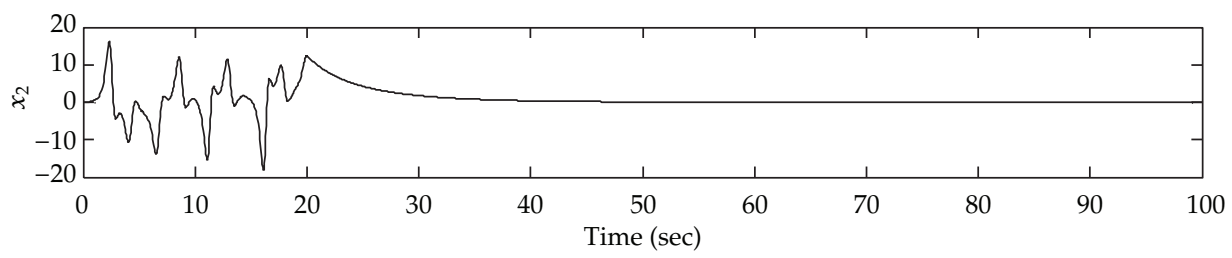

(b)

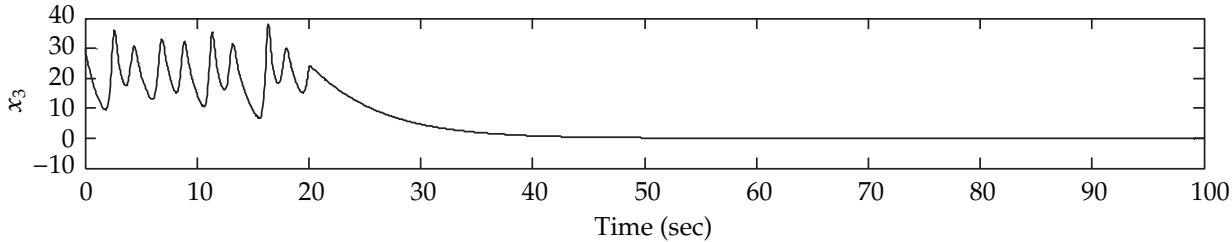

(c)

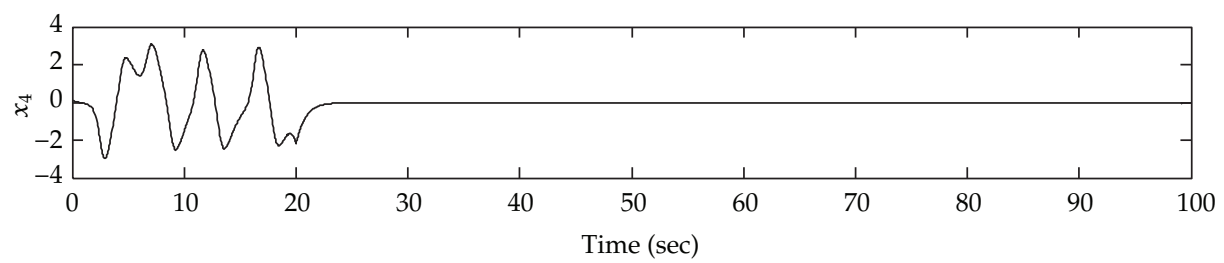

(d)

Figure 2: The state responses of the controlled 4D Lorenz-Stenflo chaotic system.

where

$$
\begin{aligned}
& x(t)=\left[\begin{array}{llll}
x_{1}(t) & x_{2}(t) & x_{3}(t) & x_{4}(t)
\end{array}\right]^{T}, \quad A_{1}=\left[\begin{array}{cccc}
-1 & 1 & 0 & 1.5 \\
26 & -1 & 7 & 0 \\
0 & -7 & -0.7 & 0 \\
-1 & 0 & 0 & -1
\end{array}\right], \\
& A_{2}=\left[\begin{array}{cccc}
-1 & 1 & 0 & 1.5 \\
26 & -1 & -7 & 0 \\
0 & 7 & -0.7 & 0 \\
-1 & 0 & 0 & -1
\end{array}\right], \quad B_{1}=\left[\begin{array}{l}
1 \\
0 \\
0 \\
0
\end{array}\right], \quad B_{2}=\left[\begin{array}{l}
1 \\
0 \\
0 \\
1
\end{array}\right] \text {. }
\end{aligned}
$$

By the theorem, the stabilizing fuzzy control gains are given by $K_{1}=K_{2}=[61.3924 .857-$ 0.137 4.026]. 
Consequently, the minimal guaranteed cost is $J^{*}=6.26 \times 10^{-11}$. The simulation results with initial conditions $x(0)=\left[\begin{array}{llll}0.1 & 0.1 & 30 & 0.1\end{array}\right]^{T}$ are shown in Figures 1 and 2. The chaotic attractor of $4 \mathrm{D}$ Lorenz-Stenflo system is given in Figure 1. The system state responses trajectory of controller design is shown in Figure 2. When $t=20 \mathrm{sec}$, it is obvious that the feedback control gain can guarantee stable of $4 \mathrm{D}$ Lorenz-Stenflo systems. From the simulation results, it is shown that the proposed controller works well to guarantee stable.

\section{Conclusion}

This paper has presented the solutions to the guaranteed cost control of chaos problem via the Takagi-Sugeno fuzzy control for 4D Lorenz-Stenflo system. Based on Lyapunov stability theory and LMI technique, the guaranteed cost control gains can be easily obtained through a convex optimization problem. Finally, a numerical example shows the validity and superiority of the developed result.

\section{References}

[1] M. S. H. Chowdhury and I. Hashim, "Application of multistage homotopy-perturbation method for the solutions of the Chen system," Nonlinear Analysis, vol. 10, no. 1, pp. 381-391, 2009.

[2] U. E. Vincent, "Synchronization of identical and non-identical 4-D chaotic systems using active control," Chaos, Solitons and Fractals, vol. 37, no. 4, pp. 1065-1075, 2008.

[3] E. Kurt and M. Canturk, "Chaotic dynamics of resistively coupled DC-driven distinct Josephson junctions and the effects of circuit parameters," Physica D, vol. 238, pp. 2229-2237, 2009.

[4] T. Gao, Q. Gu, and S. Emmanuel, "A novel image authentication scheme based on hyper-chaotic cell neural network," Chaos, Solitons \& Fractals, vol. 42, pp. 548-553, 2009.

[5] A. Loría, "Control of the new 4th-order hyper-chaotic system with one input," Communications in Nonlinear Science and Numerical Simulation, vol. 15, no. 6, pp. 1621-1630, 2010.

[6] S. Pang and Y. Liu, "A new hyperchaotic system from the Lü system and its control," Journal of Computational and Applied Mathematics, vol. 235, no. 8, pp. 2775-2789, 2011.

[7] A. Gökdoğan, M. Merdan, and A. Yildirim, "The modified algorithm for the differential transform method to solution of Genesio systems," Communications in Nonlinear Science and Numerical Simulation, vol. 17, no. 1, pp. 45-51, 2012.

[8] Y. Yu and H.-X. Li, "Adaptive hybrid projective synchronization of uncertain chaotic systems based on backstepping design," Nonlinear Analysis, vol. 12, no. 1, pp. 388-393, 2011.

[9] C. Zhu, "Feedback control methods for stabilizing unstable equilibrium points in a new chaotic system," Nonlinear Analysis, vol. 71, no. 7-8, pp. 2441-2446, 2009.

[10] J.-S. Lin and J.-J. Yan, "Adaptive synchronization for two identical generalized Lorenz chaotic systems via a single controller," Nonlinear Analysis, vol. 10, no. 2, pp. 1151-1159, 2009.

[11] H. Zhu and B. Cui, "Stabilization and synchronization of chaotic systems via intermittent control," Communications in Nonlinear Science and Numerical Simulation, vol. 15, no. 11, pp. 3577-3586, 2010.

[12] H.-T. Yau and C.-S. Shieh, "Chaos synchronization using fuzzy logic controller," Nonlinear Analysis, vol. 9, no. 4, pp. 1800-1810, 2008.

[13] A. Gökdoğan, A. Yildirim, and M. Merdan, "Solving a fractional order model of HIV infection of CD4 ${ }^{+}$T cells," Mathematical and Computer Modelling, vol. 54, no. 9-10, pp. 2132-2138, 2011.

[14] T. Takagi and M. Sugeno, "Stability and stabilizability of fuzzy-neural linear control systems," IEEE Transactions on Fuzzy Systems, vol. 3, pp. 438-447, 1995.

[15] C. Hu, H. Jiang, and Z. Teng, "General impulsive control of chaotic systems based on a TS fuzzy model," Fuzzy Sets and Systems, vol. 174, pp. 66-82, 2011.

[16] F. Liu, M. Wu, Y. He, and R. Yokoyama, "New delay-dependent stability criteria for T-S fuzzy systems with time-varying delay," Fuzzy Sets and Systems, vol. 161, no. 15, pp. 2033-2042, 2010.

[17] W. Y. Wang, Y. H. Chien, Y. G. Leu, and T. T. Lee, "Adaptive T-S fuzzy-neural modeling and control for general MIMO unknown nonaffine nonlinear systems using projection update laws," Automatica, vol. 46, pp. 852-863, 2010. 
[18] R. Rajesh and M. R. Kaimal, “T-S fuzzy model with nonlinear consequence and PDC controller for a class of nonlinear control systems," Applied Soft Computing, vol. 7, pp. 772-782, 2007.

[19] S. Boyd, L. El Ghaoui, E. Feron, and V. Balakrishnan, Linear Matrix Inequalities in System and Control Theory, vol. 15, Society for Industrial and Applied Mathematics, Philadelphia, Pa, USA, 1994. 


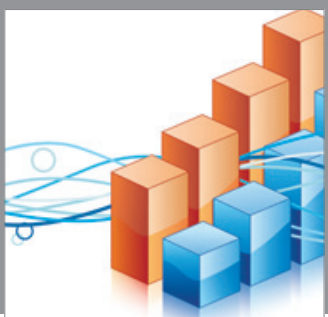

Advances in

Operations Research

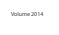

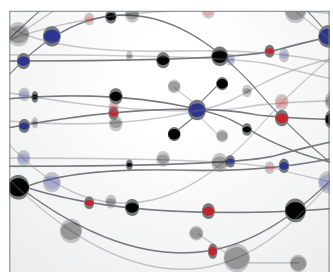

\section{The Scientific} World Journal
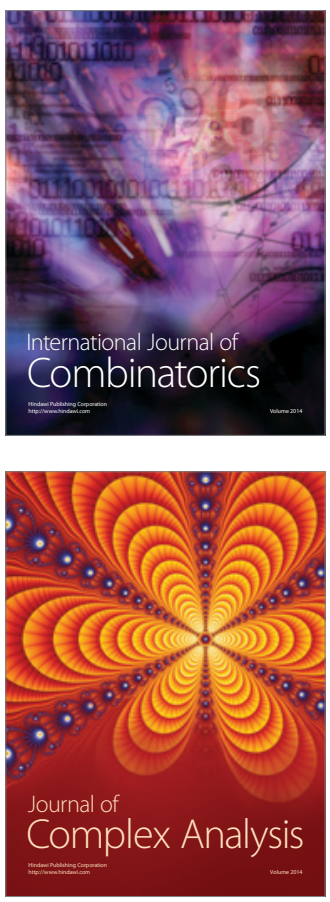

International Journal of

Mathematics and

Mathematical

Sciences
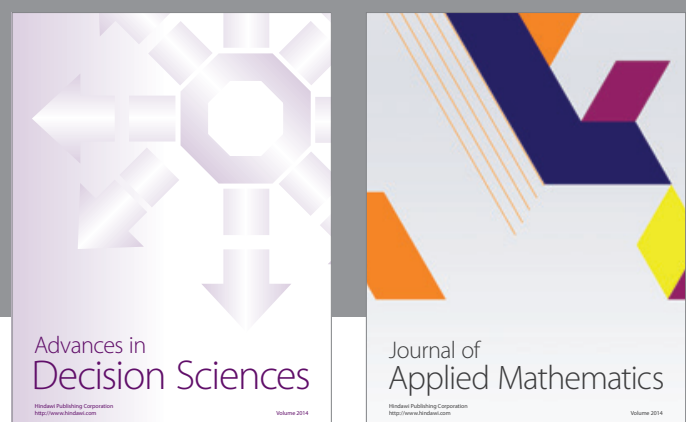

Journal of

Applied Mathematics
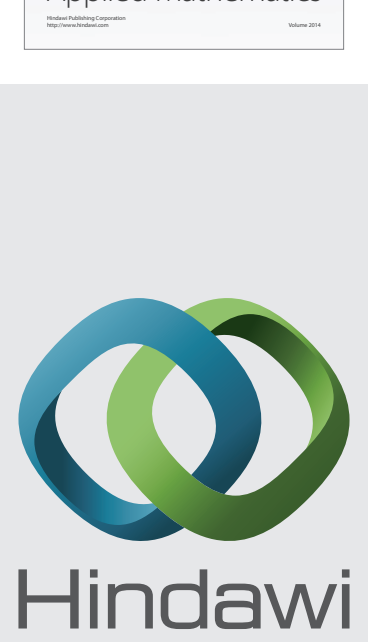

Submit your manuscripts at http://www.hindawi.com
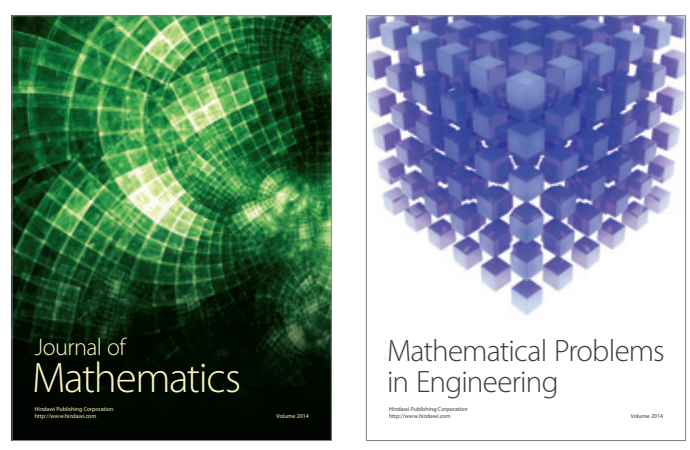

Mathematical Problems in Engineering
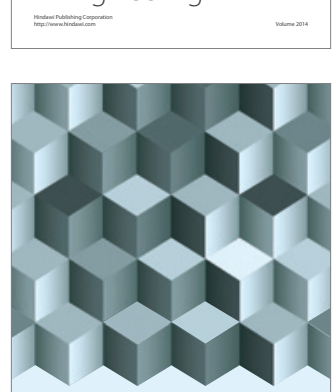

Journal of

Function Spaces
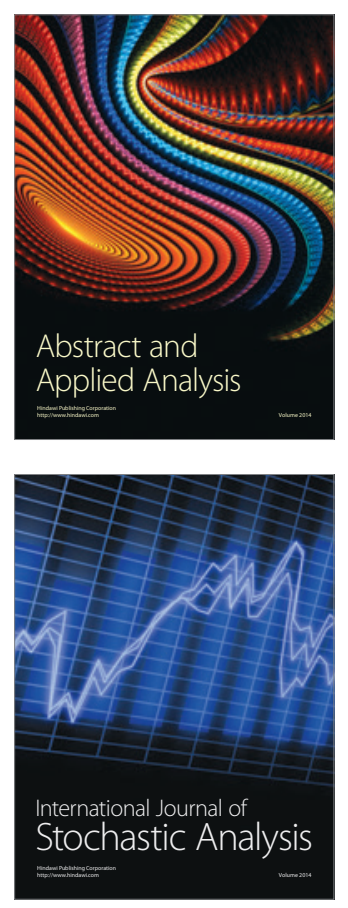

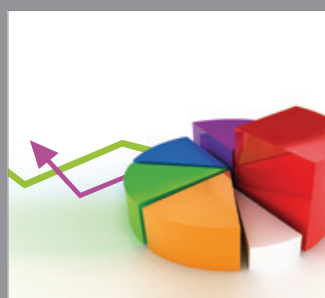

ournal of

Probability and Statistics

Promensencen
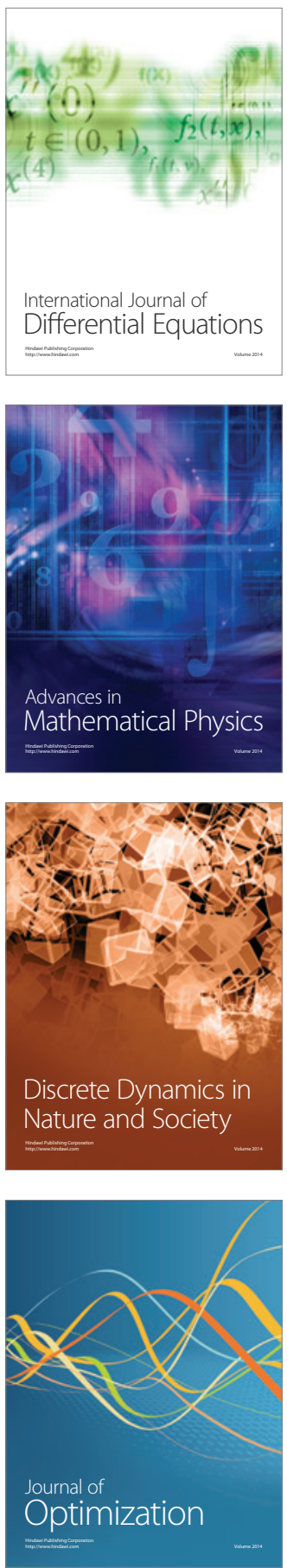